\title{
Laparoscopic treatment of an intrauterine device mislocated in the abdominal region
}

\author{
Jakub Sliwa, Tomasz Fuchs, Anna Kryza-Ottou, Mariusz Zimmer \\ Medical University of Wroclaw, Departament of Obstetrics and Gynecology, Wroclaw, Poland
}

A 41-year-old patient (gravida 2, para 2) after two cesarean sections $(2007,2010)$ due to ophthalmologic indications was admitted for a diagnostic laparoscopy to search for an intrauterine device (IUD) that had relocated to the abdominal cavity. The woman was asymptomatic; inflammation markers were normal. Previous hysteroscopy had not found any defect of the cesarean section scar but confirmed adhesions in the uterine cavity. The IUD with a levonorgestrel (Mirena) was implemented in November 2018. After 3 months, the IUD was not visible in the uterine cavity on ultrasound. An abdominal X-ray showed a perforated IUD in the right iliac fossa (Fig. 1A). On laparoscopy, the IUD was found to be ingrown in the greater omentum next to the intestinal wall (Fig. 1B-C). The insert was separated from the adhesions with the omentum without damaging the intestine and removed through the lateral trocar. No perforation within the uterus nor damage to abdominal organs were found. The postoperative course was uneventful. The patient was discharged home on the second day post-procedure.

Uterine perforation is a complication of IUD implementation with a frequency of 0-1.3 per 1000 applications. It is associated with the risk of damaging intestines and other abdominal organs by the perforated insert [1]. This case is unique because the IUD was inserted 6 months earlier and the patient did not feel any discomfort during this period. The UID relocation was found accidentally during the follow-up visit.

The relocated UID must be removed due to the risk of damaging abdominal organs [2]. The overall rate of successful laparoscopic procedures for UID removal in cases of uterine perforation reaches $64 \%$ of UIDs [3]. Removal of a lost insert may require conversion to a laparotomy, especially when no perforation is visible within the uterus or adhesions and intestinal damage occur. In this case, the IUD removal was performed laparoscopically.

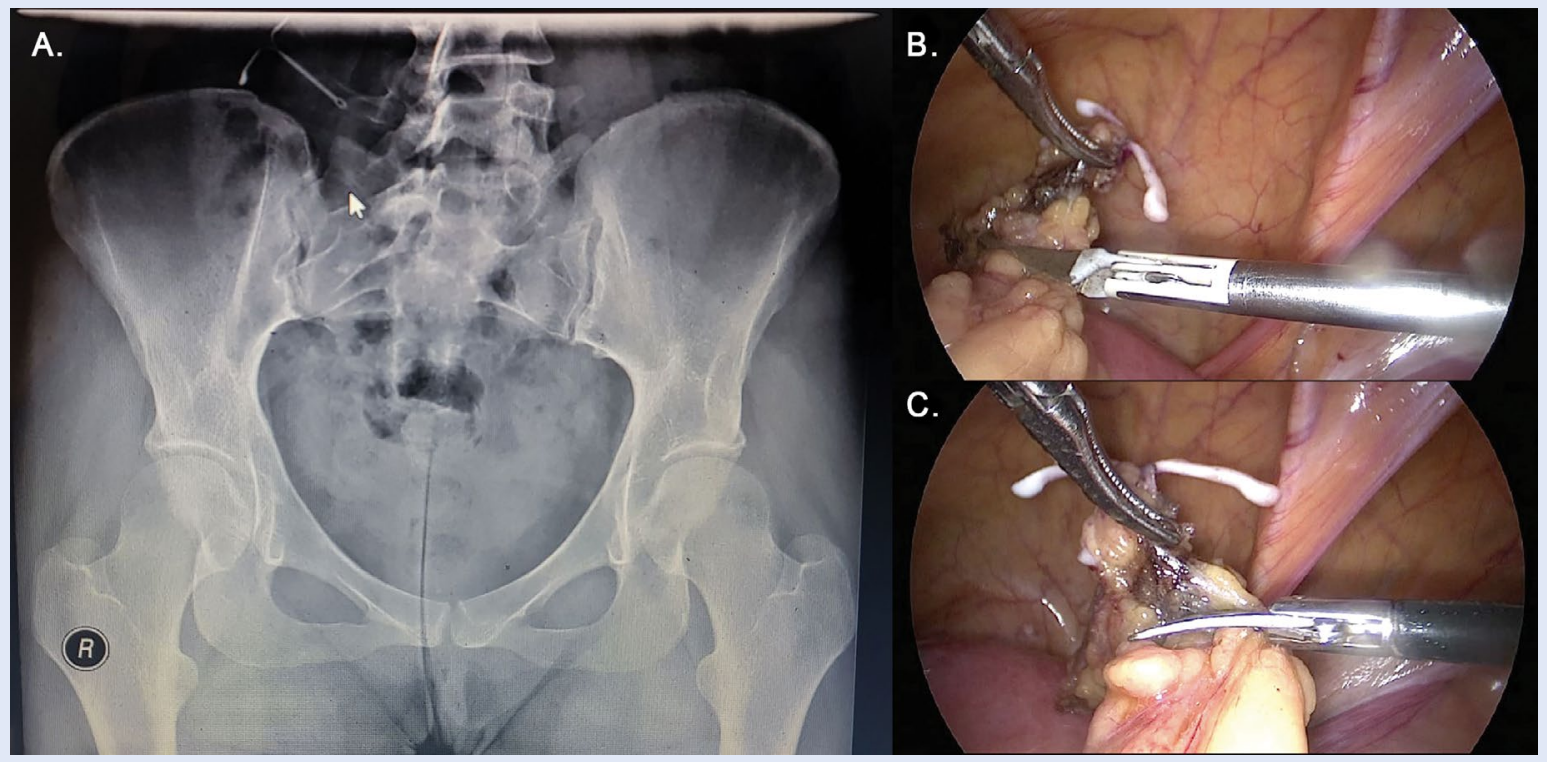

Figure 1. Perforated intrauterine device: $A$ - the device marked with an arrow in the right iliac fossa; an abdominal X-ray; $B$ — laparoscopic image of a perforated intrauterine device ingrown in the greater omentum next to the intestinal wall, $C$ - laparoscopic removal of the intrauterine device

\section{REFERENCES}

1. Gardyszewska A, Niewiadomska-Kowalczyk M, Szymańska B, et al. [Extrauterine mislocated IUD]. Ginekol Pol. 2009; 80(12): 942-945, indexed in Pubmed: 20120941.

2. Owens L, Rattner A, Burke A, et al. Laparoscopic Management of an Unusual Intrauterine Device Perforation After Postplacental Insertion. J Minim Invasive Gynecol. 2016; 23(2): 156-157, doi: 10.1016/j.jmig.2015.06.011, indexed in Pubmed: 26093184.

3. Gill RS, Mok D, Hudson M, et al. Laparoscopic removal of an intra-abdominal intrauterine device: case and systematic review. Contraception. 2012; 85(1): 15-18, doi: 10.1016/j.contraception.2011.04.015, indexed in Pubmed: 22067801. 